\title{
Simultaneous optimization of colloidal stability and
}

\section{interfacial charge transfer efficiency in photocataly-}

\section{tic Pt/CdS nanocrystals}

Wei Li**, Jonathan R. Lee, Frank Jäckel*

Department of Physics and Stephenson Institute for Renewable Energy, The University of Liverpool, Chadwick Building, Peach Street, Liverpool, L69 7ZF, UK.

† Present address: Chemical Engineering \& Applied Chemistry, European Bioenergy Research Institute and Aston Materials Centre, Aston University, Aston Triangle, Birmingham B4 7ET, UK

ABSTRACT Colloidal stability and efficient interfacial charge transfer in semiconductor nanocrystals are of great importance for photocatalytic applications in aqueous solution since they provide long-term functionality and high photocatalytic activity, respectively. However, colloidal stability and interfacial charge transfer efficiency are difficult to optimize simultaneously since the ligand layer often acts as both a shell stabilizing the nanocrystals in colloidal suspension and a barrier reducing the efficiency of interfacial charge transfer. Here we show that for cysteinecoated, Pt-decorated CdS nanocrystals and $\mathrm{Na}_{2} \mathrm{SO}_{3}$ as hole scavenger, triethanolamine (TEOA) replaces the original cysteine ligands in-situ and prolongs the highly efficient and steady $\mathrm{H}_{2}$ 
evolution period by more than a factor of 10 . It is shown that $\mathrm{Na}_{2} \mathrm{SO}_{3}$ is consumed during $\mathrm{H}_{2}$ generation while TEOA makes no significant contribution to the $\mathrm{H}_{2}$ generation. An apparent quantum yield of $31.5 \%$, a turnover frequency of $0.11 \mathrm{H}_{2} / \mathrm{Pt} / \mathrm{s}$ and an interfacial charge transfer rate faster than 0.3 ps were achieved in the TEOA stabilized system. The short length, branched structure and weak binding of TEOA to CdS as well as sufficient free TEOA in the solution are the keys to enhancing colloidal stability and maintaining efficient interfacial charge transfer at the same time. Additionally, TEOA is commercially available and cheap and we anticipate that this approach can be widely applied in many photocatalytic applications involving colloidal nanocrystals.

KEYWORDS aggregation free, electron transfer, hole scavenging, transient absorption spectroscopy, photocatalysis, water splitting, solar fuels. 


\section{INTRODUCTION}

Colloidal nanocrystals (NCs) have attracted extensive scientific and industrial interests due to their unique optical and electronic properties, ease of functionalization and low cost synthesis, which makes them attractive for applications in various fields from energy conversion to biological labelling. ${ }^{1,2}$ Particularly, colloidal co-catalyst-decorated semiconductor NCs have recently been increasingly investigated for photocatalytic $\mathrm{H}_{2}$ generation. ${ }^{3-7}$ These water soluble colloidal NCs are most commonly stabilized by chemically attaching a monolayer of hydrophilic thiols to the atoms on the surface of NCs. Colloidally stable (i.e. aggregation-free) NCs in aqueous solution are necessary to ensure long term functionality and efficiency. ${ }^{8,9}$

In photocatalytic $\mathrm{H}_{2}$ production, interfacial transfer of photogenerated electrons and holes from the photocatalyst to water and hole scavenging molecules in the aqueous phase, respectively, are key steps. ${ }^{5,10-13}$ Therefore, it is a dilemma that photocatalytic NCs need a protective (thick or dense) shell to be colloidally stable while this shell often prevents efficient interfacial charge transfer by hindering surface access. ${ }^{14-17}$

Short and small hydrophilic thiols (cysteine, MPA, etc.) are widely used as ligands for photocatalytic NCs. ${ }^{6,18}$ However, they can be photooxidized and partially removed as soon as the material is illuminated. ${ }^{19,20}$ In this case, the thin and broken ligand shell temporarily stabilizes the nanocrystal and allows interfacial charge transfer. However, due to the continuous removal of ligands, aggregation of NCs eventually occurs and consequently reduces photocatalytic efficiency. This metastable period normally lasts from several minutes up to hours depending on many factors such as illumination intensity, nanocrystal size, type of ligands, $\mathrm{pH}$ and ionic strength of the solution, and stirring rate. ${ }^{19,21}$ 
The photooxidation and removal of thiols have been shown to be the initial and key steps for the aggregation process. ${ }^{19}$ Successively supplied thiols or closely packed branched thiol ligands have been used to provide enhanced stability, but their close packing blocks efficient interfacial charge transfer and reduces photocatalytic activity. ${ }^{22}$ Here we show that by employing a shorter, less strongly bound and branched ligand such as triethanolamine (TEOA) and providing it successively, colloidal stability and efficient interfacial charge transfer can be achieved simultaneously.

We investigate $\mathrm{H}_{2}$ generation and employ ultrafast transient absorption spectroscopy to clearly demonstrate that TEOA is not only able to colloidally stabilize NCs but also to allow for efficient interfacial charge transfer. For Pt-decorated $\mathrm{CdS}(\mathrm{Pt} / \mathrm{CdS}) \mathrm{NCs}$ and $\mathrm{Na}_{2} \mathrm{SO}_{3}$ as a hole scavenger, TEOA prolonged the highly efficient and steady $\mathrm{H}_{2}$ evolution period by more than a factor of 10 . It is shown that $\mathrm{Na}_{2} \mathrm{SO}_{3}$ is consumed during $\mathrm{H}_{2}$ generation while TEOA makes no significant contribution to the $\mathrm{H}_{2}$ generation. An apparent quantum yield of $31.5 \%$, a turnover frequency of $0.11 \mathrm{H}_{2} / \mathrm{Pt} / \mathrm{s}$ and an interfacial charge transfer rate faster than $0.3 \mathrm{ps}$ were achieved in the presence of TEOA. Initial removal of the cysteine surface ligands on the as-prepared Pt/CdS NCs and their simultaneous replacement by TEOA are critical steps for the ensuing stable and efficient $\mathrm{H}_{2}$ evolution. The short and branched geometry of TEOA, its weak binding to CdS and the availability of free TEOA in the solution are the keys for the improved colloidal stability as well as high efficiency of the system. Since TEOA is commercially available and very cheap, this approach can be widely applied in many photocatalytic applications involving colloidal NCs. 

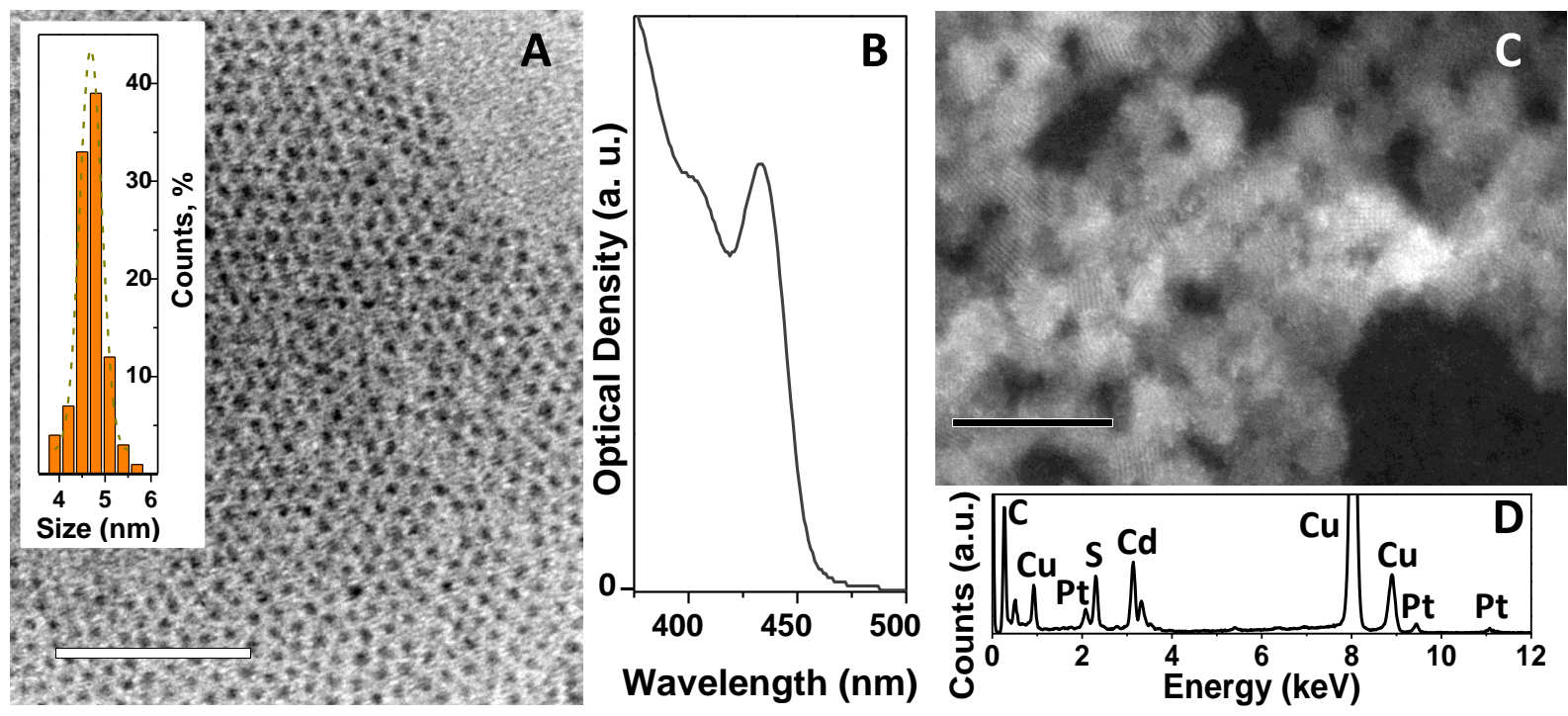

Figure 1. A) TEM image with size distribution (inset) and B) Extinction spectrum of CdS NCs with an average size of $4.7 \mathrm{~nm}$ and the first excitonic peak at $437 \mathrm{~nm}$. C) HAADF-STEM image and D) EDX analysis of Pt/CdS NCs on carbon films coated copper grids.

Figure 1 illustrates the materials employed. Spherical CdS NCs were synthesized in oleic acid/octadecene, phase transferred into aqueous solution with cysteine ligands and decorated with sub-nm Pt clusters using photodeposition. Figure 1A displays a TEM image of the as-synthesized CdS NCs with an average size of $4.7 \mathrm{~nm}$ and a narrow size distribution with a standard deviation of $0.2 \mathrm{~nm}$. Figure 1B displays the extinction spectra of the CdS NCs. The size of CdS NCs is calculated ${ }^{23}$ to be $4.7 \mathrm{~nm}$ from peak position of the first excitonic transition at $437 \mathrm{~nm}$ in agreement with the average size determined from the TEM images. The narrow and sharp absorption peak further illustrates the narrow size distribution of the NCs. Elemental composition of the Pt/CdS NCs was determined with Inductively Coupled Plasma - Optical Emission Spectrometry (ICPOES) and a Pt content of $\sim 16 \mathrm{wt} \%$ was found. Element-sensitive high-angle annular dark field scanning TEM (HAADF-STEM) coupled with energy-dispersive x-ray (EDX) analysis has been 
employed to check the morphology of Pt clusters photodeposited on CdS NCs. Representative results are shown in Figure 1C and 1D. The EDX result confirms decoration of the NCs was around $13 \mathrm{wt} \% \mathrm{Pt}$, showing good agreement with the ICP-OES measurement. HAADF-STEM images of $\mathrm{Pt} / \mathrm{CdS}$ demonstrate that the photodeposited Pt clusters are well dispersed and have a very small size beyond the detection limit of the instrument. Crystal lattice separations were measured from Figure $1 \mathrm{C}$ being consistent with a hexagonal wurtzite phase of the CdS NCs.

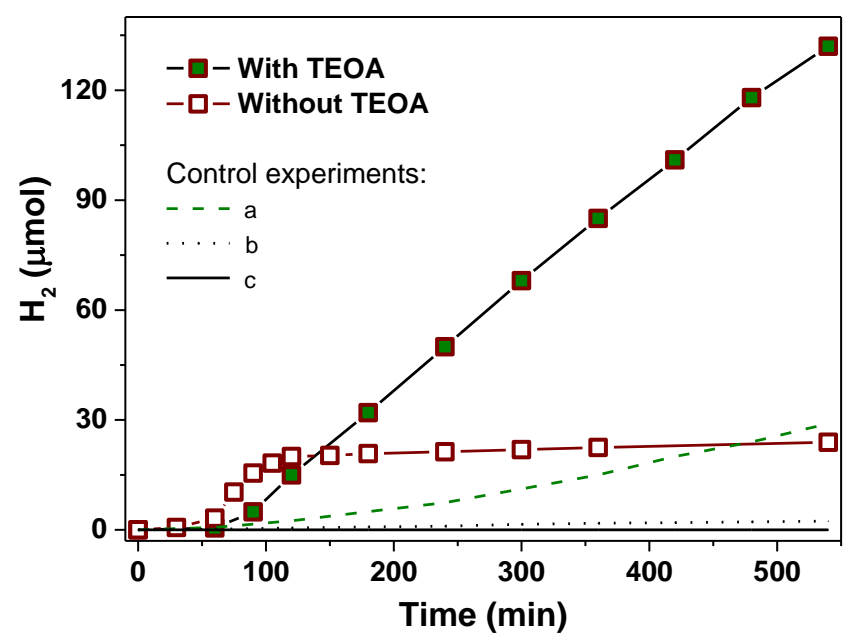

Figure 2. Photocatalytic hydrogen evolution of Pt/CdS NCs with $0.1 \mathrm{M} \mathrm{TEOA}+0.02 \mathrm{M} \mathrm{Na}_{2} \mathrm{SO}_{3}$ (i.e. TEOA/ $\mathrm{Na}_{2} \mathrm{SO}_{3}$-mixed system, solid squares) and $0.02 \mathrm{M} \mathrm{Na}_{2} \mathrm{SO}_{3}$ only (open squares). A set of control experiments is also displayed: Photocatalytic hydrogen evolution from Pt/CdS NCs with $0.1 \mathrm{M}$ TEOA only (dashed line), from $0.1 \mathrm{M} \mathrm{TEOA}+0.02 \mathrm{M} \mathrm{Na}_{2} \mathrm{SO}_{3}$ under light irradiation without $\mathrm{Pt} / \mathrm{CdS} \mathrm{NCs}$ (dot line), and from 0.1M TEOA $+0.02 \mathrm{M} \mathrm{Na}_{2} \mathrm{SO}_{3}$ with Pt/CdS NCs in dark (solid line).

Figure 2 shows our key results, namely that the period of $\mathrm{H}_{2}$ evolution by TEOA stabilized $\mathrm{Pt} / \mathrm{CdS}$ NCs in the presence of $\mathrm{Na}_{2} \mathrm{SO}_{3}$ as hole scavenger (hereafter referred to as the TEOA/Na2 $\mathrm{SO}_{3}$ mixed system) is extended by a factor of 10 compared to the $\mathrm{Na}_{2} \mathrm{SO}_{3}$ only system. At the same time the rate of $\mathrm{H}_{2}$ evolution remains the same in both systems. For the $\mathrm{Na}_{2} \mathrm{SO}_{3}$ only system a 
significant decay of the $\mathrm{H}_{2}$ evolution rate was observed after a short period of efficient $\mathrm{H}_{2}$ generation (less than $45 \mathrm{~min}, \sim 18 \mu \mathrm{mol} / \mathrm{h}$ ). The period of colloidal stability is comparable to similar systems reported previously (usually less than $100 \mathrm{~min})^{19,22}$. By adding TEOA, a steady rate of $17.5 \mu \mathrm{mol} / \mathrm{h}$ lasting up to $8 \mathrm{~h}$ of illumination was demonstrated with the same photocatalyst. Successive supply of cysteine ligands was able to maintain colloidal stability of Pt/CdS NCs but the intact cysteine shell blocked $\mathrm{H}_{2}$ evolution (data presented and discussed later) similar to the noticeable induction period of $\sim 60$ minutes visible in Fig. 2.

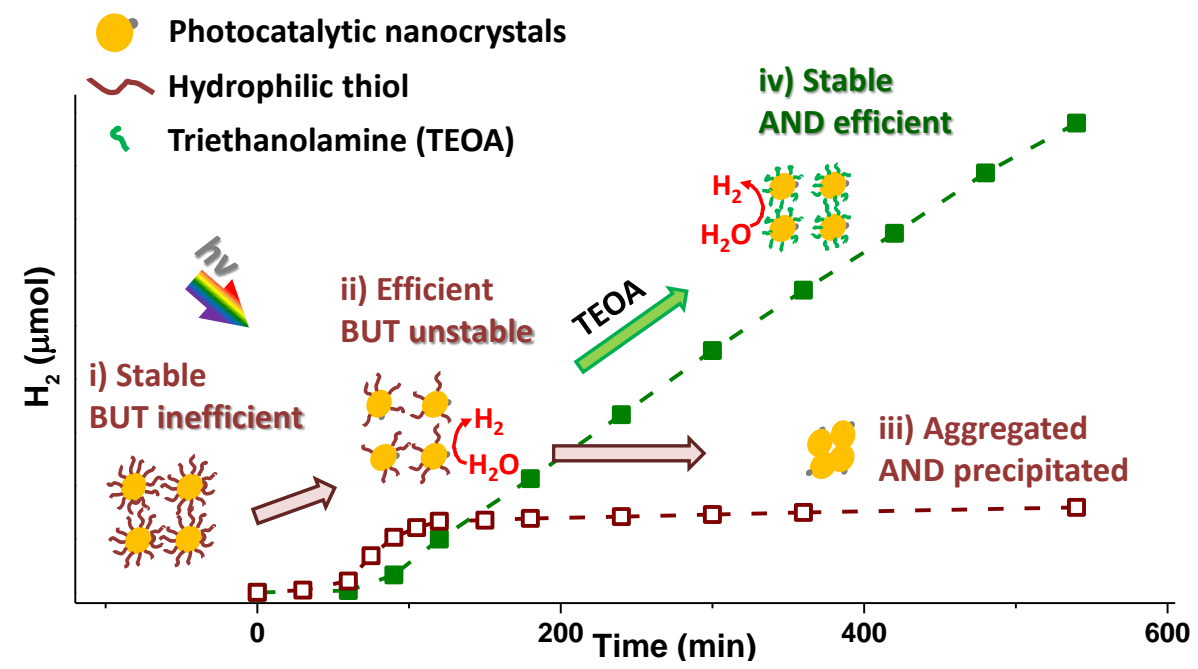

Figure 3. Proposed mechanism of the TEOA stabilized system.

$\mathrm{H}_{2}$ evolution of $\mathrm{Pt} / \mathrm{CdS}$ with TEOA only was investigated as a control experiment. A stable $\mathrm{H}_{2}$ evolution rate of $3.2 \mu \mathrm{mol} / \mathrm{h}$ was observed indicating a stable photocatalytic system. However, this rate is significantly smaller than the one observed with $\mathrm{Na}_{2} \mathrm{SO}_{3}$ as hole scavenger, consistent with a previous report by Berr et al. which found that TEOA was a less efficient hole scavenger than $\mathrm{Na}_{2} \mathrm{SO}_{3} .{ }^{10}$ In the following, we will demonstrate and discuss in detail that when using a mixture of $\mathrm{Na}_{2} \mathrm{SO}_{3}$ and TEOA, TEOA mainly acts as a ligand with a negligible contribution to hole scavenging, while $\mathrm{Na}_{2} \mathrm{SO}_{3}$ acts as the dominant hole scavenger. Whether direct photolysis of 
TEOA without photocatalyst and any dark reaction may produce $\mathrm{H}_{2}$ were tested as control experiments (Figure 1) and revealed only negligible contributions to $\mathrm{H}_{2}$ evolution. In particular, we propose a mechanism for the TEOA stabilized system as shown in Figure 3. i) initially, the intact cysteine ligand shell provides colloidal stability for $\mathrm{Pt} / \mathrm{CdS} \mathrm{NCs}$ but hinders $\mathrm{H}_{2}$ evolution resulting in an induction period; ii) when the cysteine ligands begin to photooxidize and are partially removed, the thin and broken ligand shell allows interfacial charge transfer while still temporarily stabilizing the nanocrystal. iii) due to the continuous removal of ligands, NCs aggregate if no stabilizer is added and photocatalytic efficiency is consequently reduced. iv) if TEOA is added, it serves as a substitute ligand stabilizing the NCs in situ and extending the period of efficient $\mathrm{H}_{2}$ evolution.

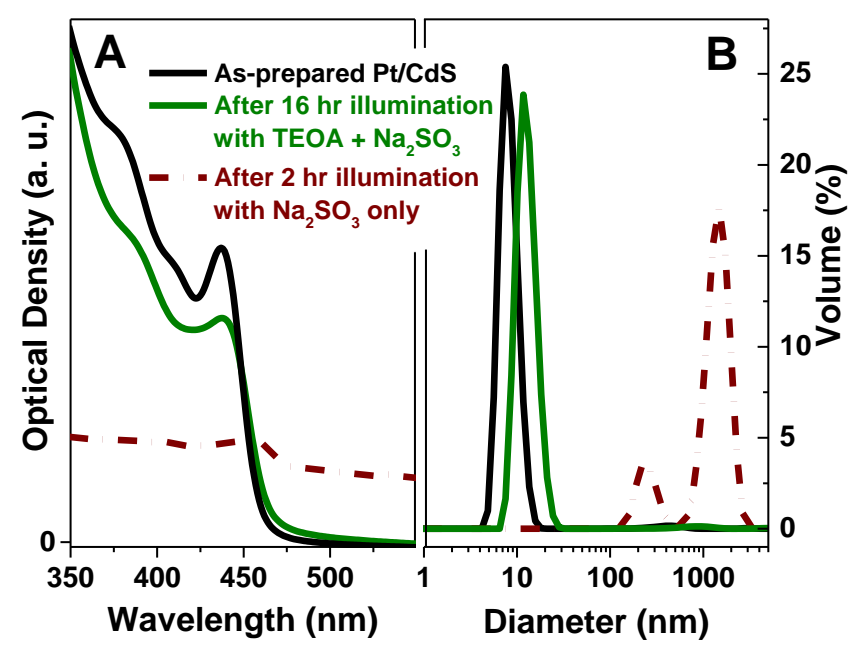

Figure 4. A) Extinction spectra and B) hydrodynamic diameter from dynamic light scattering measurements of as-prepared cysteine capped Pt/CdS NCs (black solid line), Pt/CdS NCs in the TEOA/ $\mathrm{Na}_{2} \mathrm{SO}_{3}$-mixed system after $16 \mathrm{~h}$ of $\mathrm{H}_{2}$ evolution (green solid line) and $\mathrm{Pt} / \mathrm{CdS} \mathrm{NCs}$ in the photocatalytic system with $\mathrm{Na}_{2} \mathrm{SO}_{3}$ only after $2 \mathrm{~h}$ of $\mathrm{H}_{2}$ evolution (red dot line).

We now turn to the detailed discussion of this model and start with the critical role of TEOA in the TEOA/Na2 $\mathrm{SO}_{3}$ mixed system. Firstly, we will show how TEOA replaces the original cysteine 
ligands and stabilizes the NCs and, secondly, we provide evidence from transient absorption spectroscopy (TAS) measurements that the efficiency of the interfacial charge transfer is maintained.

Figure 4 presents extinction spectra and hydrodynamic diameter distributions of Pt/CdS indicating different colloidal stability of the photocatalytic systems with and without TEOA under a range of conditions. They provide evidence that the presence of TEOA increases colloidal stability and prevents aggregation. In particular, as-prepared cysteine capped Pt/CdS NCs can be well dispersed in water and form a clear, yellowish and stable aqueous colloid showing very similar extinction properties as the CdS NCs in Figure 1B. The TEOA/ $\mathrm{Na}_{2} \mathrm{SO}_{3}$-mixed system has a similar extinction spectrum with only $1 \mathrm{~nm}$ red-shift, $\sim 20 \%$ reduced optical density and a slight broadening of the

first excitonic transition after $16 \mathrm{~h}$ of $\mathrm{H}_{2}$ evolution. The hydrodynamic diameters distributions of these two samples (before and after $16 \mathrm{~h}$ of $\mathrm{H}_{2}$ evolution) are centered at $7.5 \mathrm{~nm}$ and $11 \mathrm{~nm}$, respectively, and are thus very similar. This indicates that the TEOA/Na $\mathrm{SO}_{3}$-mixed system is colloidally stable over that period. However, in the photocatalytic systems with $\mathrm{Na}_{2} \mathrm{SO}_{3}$ only, both hydrodynamic diameter distribution and extinction spectrum demonstrate that the NCs were heavily aggregated within $2 \mathrm{~h}$ of illumination. This can be seen from the vanishing and broadening first excitonic peak and a significantly larger hydrodynamic radius in Fig. 4A and Fig. 4B, respectively. 

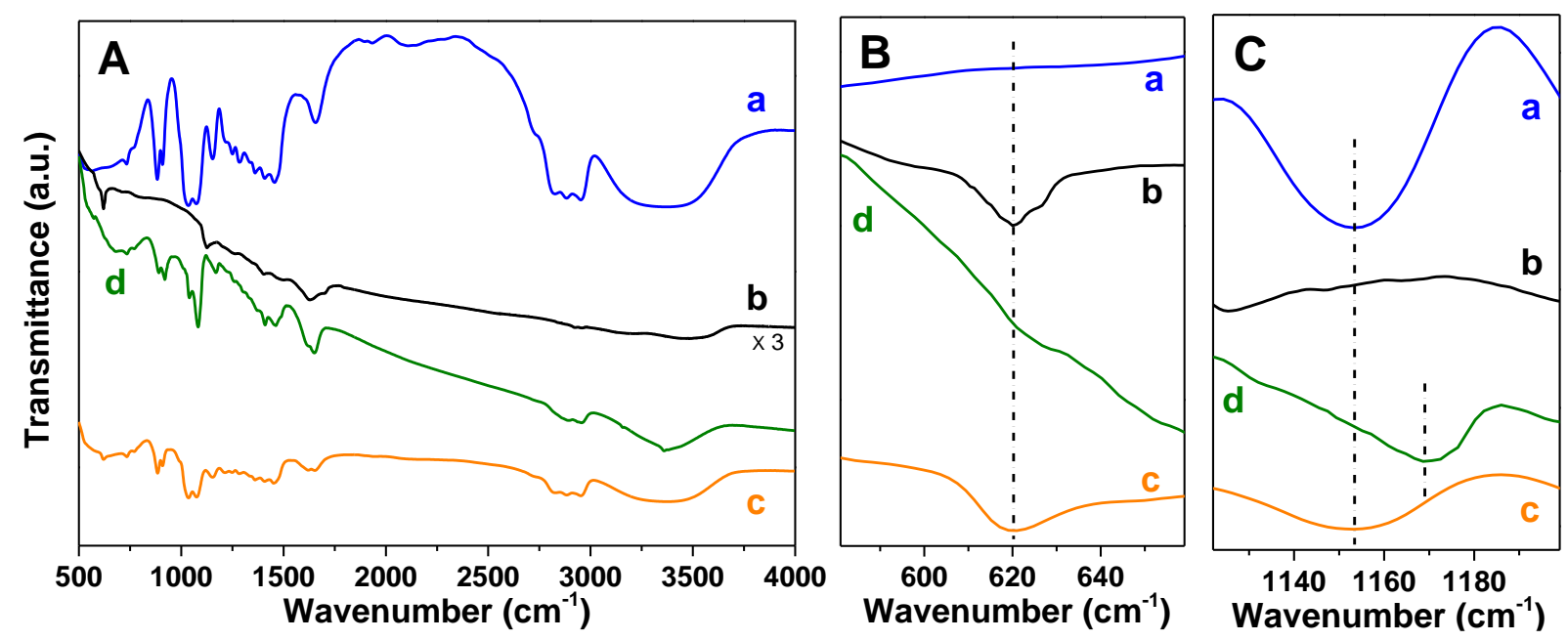

Figure 5. A) FT-IR spectra of free TEOA (a), as prepared cysteine-capped Pt/CdS (b), recovered $\mathrm{Pt} / \mathrm{CdS}$ from TEOA/Na2 $\mathrm{SO}_{3}$-mixed system before (c) and after $1 \mathrm{~h}$ of illumination (d). B) and C) are zoom-ins of the spectra in A) around wavenumber of $620 \mathrm{~cm}^{-1}$ and $1155 \mathrm{~cm}^{-1}$, respectively. Spectrum (b) in A) has been multiplied by a factor of 3 for clarity.

Figure 5 displays FT-IR spectra of free TEOA (a), as prepared cysteine capped Pt/CdS (b), recovered $\mathrm{Pt} / \mathrm{CdS}$ from TEOA/Na $\mathrm{SO}_{3}$-mixed system before (c) and after $1 \mathrm{~h}$ of illumination (d) which provide evidence that TEOA provides colloidal stability by replacing the original cysteine ligands. FT-IR spectroscopy is routinely used to monitor ligand exchange on NCs. TEOA absorption bands occur at $1037 \mathrm{~cm}^{-1}(v(\mathrm{C}-\mathrm{O})), 1075 \mathrm{~cm}^{-1}(v(\mathrm{C}-\mathrm{C})), 1155 \mathrm{~cm}^{-1}(v(\mathrm{C}-\mathrm{N})), 1350-1470$ $\mathrm{cm}^{-1}\left(\delta\left(\mathrm{CH}_{2}\right)\right), 2800-3000 \mathrm{~cm}^{-1}\left(v\left(\mathrm{CH}_{2}\right)\right)$, and $3355 \mathrm{~cm}^{-1}(v(\mathrm{OH})) .{ }^{24}$ IR absorption bands of cysteine occur at $620 \mathrm{~cm}^{-1}\left(\delta\left(\mathrm{COO}^{-}\right)\right), 1125 \mathrm{~cm}^{-1}\left(\rho\left(\mathrm{NH}_{3}{ }^{+}\right)\right), 1350-1470 \mathrm{~cm}^{-1}\left(\delta\left(\mathrm{CH}_{2}\right)\right)$ and 1618 $\mathrm{cm}^{-1}\left(\delta\left(\mathrm{NH}_{3}{ }^{+}\right)\right) .{ }^{25}$ The recovered $\mathrm{Pt} / \mathrm{CdS}$ before illumination (sample c) shows a fingerprint absorption band of cysteine at $620 \mathrm{~cm}^{-1}\left(\delta\left(\mathrm{COO}^{-}\right)\right)$with all of the other IR absorption bands contributed by TEOA. After an hour illumination (sample d), there is no detectable IR absorption of cysteine. All the observed bands can be assigned to TEOA. Importantly, a shift from $1155 \mathrm{~cm}^{-}$ ${ }^{1}$ (samples a and c) to $1170 \mathrm{~cm}^{-1}$ (sample d) of $v(\mathrm{C}-\mathrm{N})$ of TEOA is clearly demonstrated. This shift 
was observed in previous IR investigations on free and adsorbed TEOA, where the former was assigned to free TEOA and the latter was observed in adsorbed TEOA. ${ }^{26}$ These FT-IR analyses suggest that $\mathrm{Pt} / \mathrm{CdS}$ NCs were capped and stabilized by cysteine before illumination, but that during illumination cysteine ligands were removed and free TEOA molecules adsorbed to the newly vacant $\mathrm{CdS}$ surface sites via the amine.

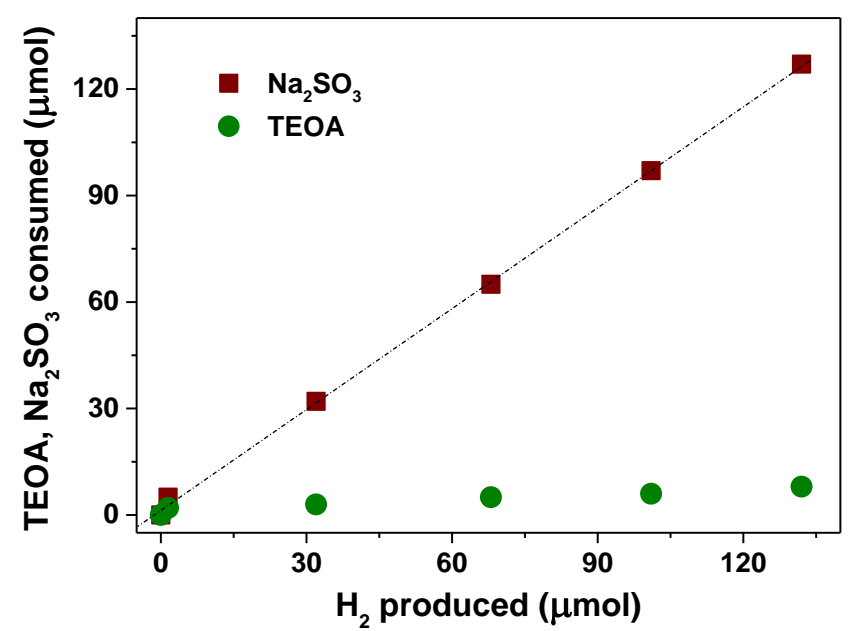

Figure 6. TEOA and $\mathrm{Na}_{2} \mathrm{SO}_{3}$ consumption, respectively, during $\mathrm{H}_{2}$ evolution in the TEOA/Na2 $\mathrm{SO}_{3}$-mixed system.

Figure 6 displays the consumption of TEOA and $\mathrm{Na}_{2} \mathrm{SO}_{3}$ during the $\mathrm{H}_{2}$ evolution process, providing evidence that during $\mathrm{H}_{2}$ generation $\mathrm{Na}_{2} \mathrm{SO}_{3}$ acts as the hole scavenger, not TEOA. In Fig. $6132 \mu \mathrm{mol} \mathrm{H}_{2}$ was produced (i.e. $264 \mu \mathrm{mol}$ electrons were consumed), consumption of $\mathrm{Na}_{2} \mathrm{SO}_{3}$ was found to be $127 \mu \mathrm{mol}$ in total and linearly correlated with the amount of $\mathrm{H}_{2}$ generated. In the hole scavenging reaction $\mathrm{SO}_{3}{ }^{2-}+2 \mathrm{OH}^{-}+2 \mathrm{~h}^{+} \rightarrow \mathrm{SO}_{4}{ }^{2-}+\mathrm{H}_{2} \mathrm{O}, 127 \mu \mathrm{mol} \mathrm{Na} \mathrm{SO}_{3}$ can scavenge $254 \mu \mathrm{mol}$ holes. In the whole process, less than $10 \mu \mathrm{mol}$ TEOA was used (including $\sim 2$ $\mu$ mol that estimated to be adsorbed onto the $0.3 \mu \mathrm{M} 4.7 \mathrm{~nm}$ NCs surface, assuming TEOA coverage of $4 \mathrm{~nm}^{-22}$ ). Therefore, it can be concluded that $\mathrm{Na}_{2} \mathrm{SO}_{3}$ oxidation accounted for more than $95 \%$ 
of $\mathrm{H}_{2}$ production, while TEOA contributed less than 5\%. This clearly demonstrates that TEOA does not contribute significantly to the hole scavenging in the TEOA/ $\mathrm{Na}_{2} \mathrm{SO}_{3}$-mixed system.

This observation can be explained by the different redox potentials of TEOA and $\mathrm{Na}_{2} \mathrm{SO}_{3} \cdot \mathrm{Na}_{2} \mathrm{SO}_{3}$ has a higher oxidation potential (i.e., weaker bound electrons) at $-3.0 \mathrm{eV}$ ( $v s$. vacuum) compared with TEOA at $-3.4 \mathrm{eV} .{ }^{10}$ When they are used separately, both work as hole scavengers, however due to its lower redox potential TEOA is less efficient. When used simultaneously as in the TEOA/ $\mathrm{Na}_{2} \mathrm{SO}_{3}$-mixed system, it is thermodynamically favorable that holes transfer to the stronger hole acceptor, i.e. more weakly bound electrons. This explains why in our system $\mathrm{Na}_{2} \mathrm{SO}_{3}$ works as an efficient hole scavenger while TEOA works mainly as a stabilizer. TEOA consumption was also monitored in the TEOA-only system where it was found to be linearly correlated with $\mathrm{H}_{2}$ production (data not shown here) supporting the above attribution.

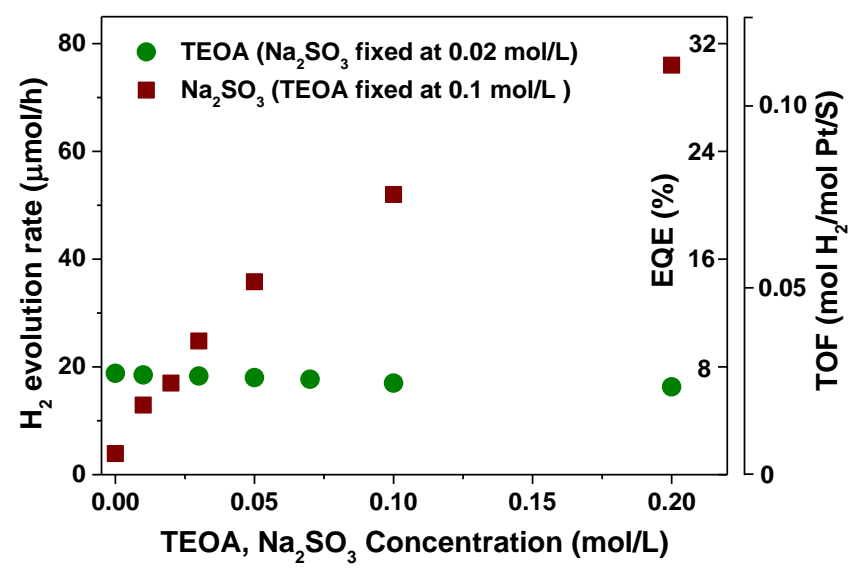

Figure 7. Dependence of $\mathrm{H}_{2}$ evolution rate, external quantum efficiency (EQE) and turn over frequency (TOF) on TEOA and $\mathrm{Na}_{2} \mathrm{SO}_{3}$ concentration, respectively.

Figure 7 displays the dependence of $\mathrm{H}_{2}$ evolution rate on TEOA and $\mathrm{Na}_{2} \mathrm{SO}_{3}$ concentrations in TEOA/Na2 $\mathrm{SO}_{3}$-mixed system, providing further evidence of the above attribution of $\mathrm{Na}_{2} \mathrm{SO}_{3}$ as hole scavenger and TEOA as stabilizer. The concentration of $\mathrm{Na}_{2} \mathrm{SO}_{3}$ significantly affects the $\mathrm{H}_{2}$ 
evolution rate with higher concentrations leading to higher efficiencies. This is the expected behavior for a hole scavenger as its concentration determines the diffusion times to reach the nanocrystal surface limiting the hole scavenging efficiency which has been shown to be the rate limiting step in related systems for $\mathrm{H}_{2}$ evolution. ${ }^{5,10-13} \mathrm{In}$ the measured range from 0 to $0.20 \mathrm{~mol} / \mathrm{L}$ of $\mathrm{Na}_{2} \mathrm{SO}_{3}$, the $\mathrm{H}_{2}$ evolution rate increases from 3 to $76 \mu \mathrm{mol} / \mathrm{h}$. It is worth emphasizing that $\mathrm{H}_{2}$ production rate of $76 \mu \mathrm{mol} / \mathrm{h}$ corresponds to high external quantum efficiency (EQE) of 31.5\% and turn over frequency (TOF) of $0.11 \mathrm{H}_{2} / \mathrm{Pt} / \mathrm{s}$. The overall photocatalytic performance exceeds those in previous reports in similar systems with EQEs in the range of $\%-10 \%{ }^{5-7,11,18}$ On the other hand, the $\mathrm{H}_{2}$ evolution rate is not significantly influenced by TEOA concentration which is expected if TEOA does not participate in the hole scavenging. Indeed, over a wide range of TEOA concentrations from 0 to $0.20 \mathrm{~mol} / \mathrm{L}, \sim 10 \%$ variation of the $\mathrm{H}_{2}$ evolution rate is observed (absolute rate in the range of $16-18 \mu \mathrm{mol} / \mathrm{h})$.

While the TEOA concentration does not affect the $\mathrm{H}_{2}$ generation efficiency significantly, it does alter the stability of the photocatalytic system dramatically. Figure 8A displays the dependence of the duration of the stable $\mathrm{H}_{2}$ evolution period on TEOA concentration. Clearly, changes in TEOA concentration affect the stability of the photocatalytic system with a notable improvement above a threshold concentration of $\sim 0.05 \mathrm{~mol} / \mathrm{L}$. As discussed above, initial illumination removes the original cysteine ligands which are replaced in-situ by TEOA. However, at low concentrations TEOA adsorption is limited by diffusion and cannot compete with particle aggregation. Thus we observe a threshold behavior reflecting that TEOA adsorption overcomes nanocrystal aggregation. 

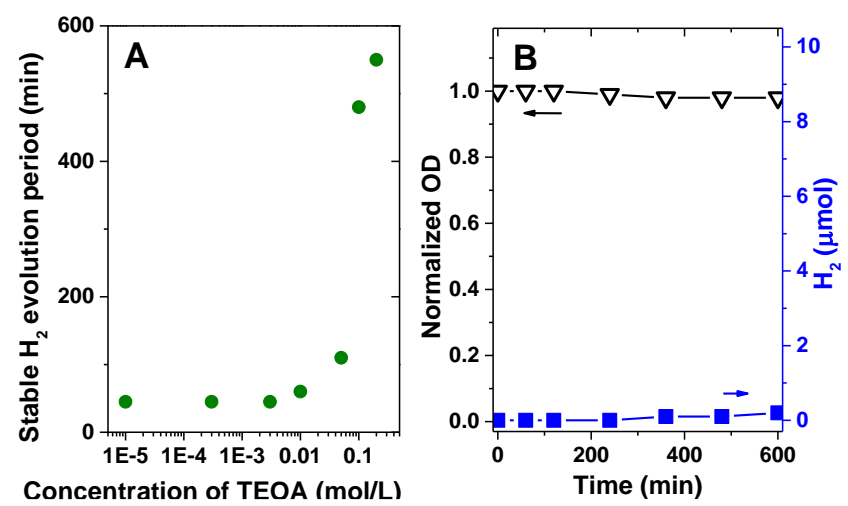

Figure 8. A) Effect of TEOA concentration on the duration of the stable $\mathrm{H}_{2}$ evolution period; B) Temporal variation of the normalized optical density (OD) at the original first excitonic absorption peak of the $\mathrm{Pt} / \mathrm{CdS} \mathrm{NCs}$ during illumination and $\mathrm{H}_{2}$ evolution, where excess free cysteine (1.0 $\mathrm{mM}$ ) was applied together with $0.02 \mathrm{M} \mathrm{Na}_{2} \mathrm{SO}_{3}$

We now verify the earlier statement that successive supply of cysteine ligands maintained colloidal stability of the Pt/CdS NCs but blocked $\mathrm{H}_{2}$ evolution. Figure 8B displays the effect of excess free cysteine on the colloidal stability by monitoring the first excitonic absorption peak of the photocatalytic solution during illumination. In a $10 \mathrm{~h}$ period, the normalized optical density at the original first excitonic absorption peak of the $\mathrm{Pt} / \mathrm{CdS} \mathrm{NCs}$ did not change significantly, demonstrating colloidal stability. In contrast, with $\mathrm{Na}_{2} \mathrm{SO}_{3}$ only (Figure 4A), aggregation of the NCs was observed within $2 \mathrm{~h}$ of illumination accompanied by a decay of the excitonic absorption peak. The improved stability can be attributed to fresh cysteine ligands adsorbing to the nanocrystal surface replacing removed/oxidized ligands. Figure $8 \mathrm{~B}$ also shows that the amount of $\mathrm{H}_{2}$ generated by cysteine stabilized $\mathrm{NCs}$ is negligible as compared to the TEOA/ $\mathrm{Na}_{2} \mathrm{SO}_{3}$-mixed system. This is further evidence that the cysteine ligand shell hinders efficient interfacial charge transfer to the hole scavenger which is critical for $\mathrm{H}_{2}$ evolution. This explanation is further supported by the observation (data not shown here) that with longer illumination time, the free cysteine molecules are slowly consumed, followed by a short period of increased $\mathrm{H}_{2}$ generation, 
which quickly terminates with nanocrystal aggregation and precipitation. The duration of the induction period (i.e. period before $\mathrm{H}_{2}$ evolution) increases with the concentration of free cysteine, accounting for the induction period of the first hour of illumination in Figure 2.

Finally, to better understand the relevant charge transfer processes in $\mathrm{Pt} / \mathrm{CdS} \mathrm{NCs}$ during photocatalytic hydrogen generation, we have conducted transient absorption spectroscopy (TAS) under realistic hydrogen generation conditions at the different stages of our photocatalytic system, i.e. as-prepared NCs, induction period, and stable $\mathrm{H}_{2}$ generation period. Spectral signatures of cadmium chalcogenide NCs and Pt decorated cadmium chalcogenide NCs have been well-studied previously and clearly assigned. ${ }^{27-29}$ The two main features discussed here are the band edge bleach upon excitation at $438 \mathrm{~nm}$ (Figure 9A), predominantly caused by electrons at the conduction band edge, and the induced absorption at $600 \mathrm{~nm}$ (Figure 9B), mainly caused by holes occupying surface traps. By monitoring the dynamics of these two signals in the TA spectra, electron and hole transfer kinetics can be studied.

Figure 9A shows that upon excitation of as-prepared $\mathrm{Pt} / \mathrm{CdS}$ NCs at $350 \mathrm{~nm}$ a band edge bleach appears within $\sim 0.5 \mathrm{ps}$ (close to the resolution of the instrument). The bleach maximum occurs at $438 \mathrm{~nm}$ and corresponds to the lowest energy exciton transition in the $\mathrm{NC}$ absorption spectrum (Figure 1B). The corresponding bleach recovery kinetics is shown in Figure 9C along with kinetics measured from the other two samples, i.e. $\mathrm{Pt} / \mathrm{CdS} \mathrm{NCs}$ during the induction period and $\mathrm{Pt} / \mathrm{CdS}$ NCs during the steady $\mathrm{H}_{2}$ evolution stage, respectively. 

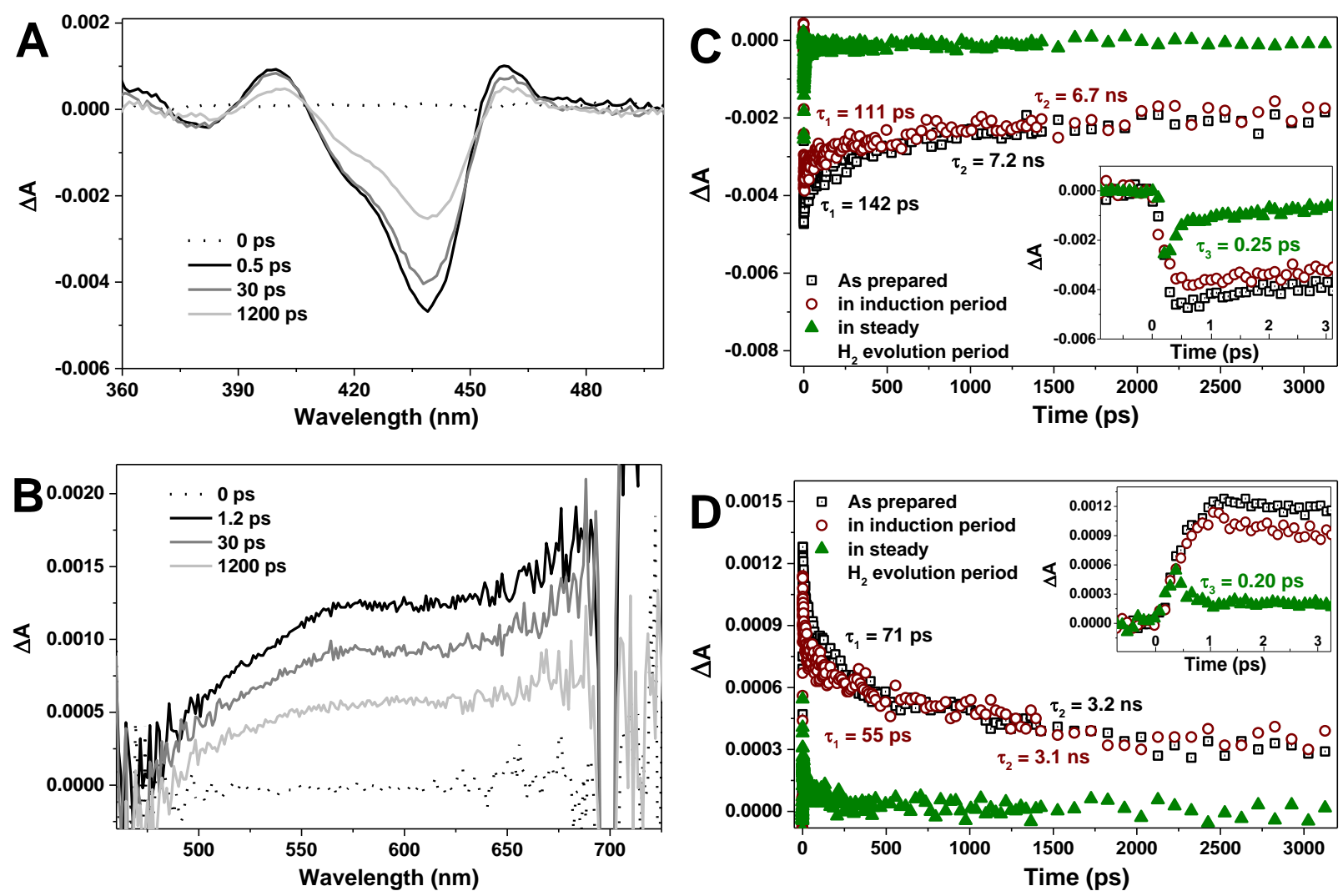

Figure 9. A) and B) Typical TAS spectra of as prepared $\mathrm{Pt} / \mathrm{CdS}$ taken at different delays following excitation; C) Dynamics of the bleaching at $438 \mathrm{~nm}$ and D) induced absorption at $600 \mathrm{~nm}$ in different $\mathrm{Pt} / \mathrm{CdS}$ samples. Insets give comparisons of early time dynamics.

Clearly, the dynamics are greatly accelerated in the steady $\mathrm{H}_{2}$ evolution period as compared to the induction period and the as-prepared NCs. The latter two show very similar dynamics with the induction period being slightly faster. Since the band edge bleaching signal is dominated by electrons at the CdS conduction band edge, this clearly shows that in the $\mathrm{H}_{2}$ evolution period electrons are transferred more quickly away from the conduction band edge. This is consistent with our previous claim that the intact ligand shell of cysteine blocks (or slows down) the charge transfer processes. The similar decays in as-prepared $\mathrm{Pt} / \mathrm{CdS} \mathrm{NCs}$ and during the induction period can be fit to biexponential decay functions (convoluted with the instrument response-function (IRF)) with time constants shown in Figure 9C. They start with a relatively fast $\sim 100$ ps relaxation, 
which is followed by a slower nanosecond decay consistent with the few nanosecond radiative lifetime of the conduction band edge electrons. This further indicates that the Pt/CdS NCs surface is well passivated by the cysteine ligands. The cysteine coverage (i.e. passivation) is reduced during the induction period, resulting in slightly faster decay. Again, this agrees with our earlier discussion that during the induction period initial ligands of cysteine start to be removed due to photo-oxidation.

The faster kinetics during the $\mathrm{H}_{2}$ evolution period can be fit using a triple exponential decay convoluted with the IRF, fixing two of the time constants to the values determined from the asprepared samples. The newly extracted time constant of $250 \pm 150 \mathrm{fs}\left(\tau_{3}\right)$ is indicative for a fast electron transfer rate. We note that these recovered $\tau_{3}$ value is very close to the time resolution (300 fs) of the TAS experiments, leading to the large errors seen in the fit value. This is also the reason for the decrease in the recorded $|\Delta \mathrm{A}|$ maximum as seen in Fig. 9C due to fast bleaching recovery quicker than instrument response functions. The actual electron transfer time may be even faster.

We now turn to the discussion of the induced absorption in the visible range from 500 to $700 \mathrm{~nm}$, which can be attributed to holes occupying surface trap states. Figure 9B shows typical induced absorptions of as-prepared Pt/CdS NCs upon excitation at $350 \mathrm{~nm}$ and Figure 9D displays decay kinetics of induced absorptions of all the same three samples studied above. Similar trends are observe for the hole dynamics as for the electron dynamics described above: i) the decays in asprepared $\mathrm{Pt} / \mathrm{CdS} \mathrm{NCs}$ and $\mathrm{Pt} / \mathrm{CdS}$ NCs during induction period show similar features with two distinct regions; ii) a faster decay is seen from $\mathrm{Pt} / \mathrm{CdS} \mathrm{NCs}$ in the steady $\mathrm{H}_{2}$ evolution stage, indicating an additional faster hole transfer channel (from NCs to hole scavengers) with a hole transfer rate of $200 \pm 150 \mathrm{fs}$ quicker than instrument response functions (300 fs). 
The overall analysis of the charge carrier dynamics from TAS is consistent with our previous discussion of the role of the ligand layer and its impact on stabilization and interfacial charge transfer. We conclude that the intact cysteine layer blocks or slows down interfacial charge transfer, leading to an induction period before $\mathrm{H}_{2}$ generation. Following initial removal of cysteine ligands, stabilization by TEOA enables efficient interfacial electron and hole transfer for steady and fast $\mathrm{H}_{2}$ production.

\section{CONCLUSIONS}

In summary, this work demonstrates that TEOA provides colloidal stability to Pt-decorated CdS NCs while enabling simultaneous efficient interfacial charge transfer for $\mathrm{H}_{2}$ generation in presence of $\mathrm{Na}_{2} \mathrm{SO}_{3}$ as hole scavenger. TEOA prolongs the highly efficient and steady $\mathrm{H}_{2}$ evolution period by more than a factor of 10 . An apparent quantum yield of $31.5 \%$, a turnover frequency of 0.11 $\mathrm{H}_{2} / \mathrm{Pt} / \mathrm{s}$ and an interfacial charge transfer rate faster than $0.3 \mathrm{ps}$ were achieved in the simultaneous presence of TEOA and $\mathrm{Na}_{2} \mathrm{SO}_{3}$. The initial removal of cysteine ligands from the as-prepared Pt/CdS NCs and their simultaneous replacement with TEOA are critical steps for the subsequent stable $\mathrm{H}_{2}$ evolution. The short, dendritic nature of TEOA, its relatively weak binding to $\mathrm{CdS}$ and its availability in the solution are the key ingredients for the colloidal stability of the system. It is worth mentioning that TEOA is commercially available and very cheap, so this approach may be widely applied in many photocatalytic applications involving colloidal NCs.

\section{MATERIALS AND METHODS}

CdS NCs synthesis. CdS NCs were synthesized in octadecene following the method of Yu and Peng. ${ }^{30}$ First, $\mathrm{CdO}(0.0576 \mathrm{~g})$, oleic acid (3.82 g), and octadecene $(18 \mathrm{~mL})$ was added in a $50-\mathrm{mL}$ three-necked flask and heated to $300{ }^{\circ} \mathrm{C}$ under $\mathrm{N}_{2}$. When solution in the flask became clear and 
colorless, another solution of sulphur $(7.2 \mathrm{mg}, 0.15 \mathrm{mmol})$ dissolved in octadecene $(9.0 \mathrm{~g})$ was quickly injected into the flask. The mixture was cooled and maintained at $250{ }^{\circ} \mathrm{C}$ for the growth of CdS NCs for $5 \mathrm{~min}$ and then it was further cooled to room temperature to stop the reaction. A mixture of hexane and ethanol $(1: 2, \mathrm{v} / \mathrm{v})$ was added to get a yellowish precipitate. The system was then centrifuged to obtain CdS NCs. Centrifugation and decantation were repeated to remove any unreacted precursors, giving the final CdS NCs monodispersed and stored in chloroform.

Phase transfer of CdS NCs and Pt deposition. Following the method of Tamang et al, ${ }^{31} \mathrm{CdS}$ NCs stored in chloroform were transferred into water by ligand exchange with $\mathrm{D}$, L-cysteine hydrochloride. Optical density of the phase transferred CdS NCs was adjusted to 1.5 at the first excitonic absorption peak. For Pt photodeposition, $10 \mathrm{~mL}$ of the phase transferred CdS NCs (OD 1.5, $\mathrm{pH} 9$ ), $65 \mathrm{mg}$ ascorbic acid, $650 \mathrm{mg}$ triethanolamine, $10 \mathrm{~mL} \mathrm{H}_{2} \mathrm{O}$ and $0.15 \mathrm{~mL} 50 \mathrm{mM}$ chloroplatinic acid hexahydrate were mixed in a $50-\mathrm{mL}$ beaker. The mixture was stirred under continuous bubbling with argon and UV excitation $\left(\sim 3.5-4 \mathrm{~mW} / \mathrm{cm}^{2}\right.$ at $\left.366 \mathrm{~nm}\right)$ from a UV lamp for $20 \mathrm{~min}$. After completing the reaction, solution was centrifuged three times in Millipore 5,000 mwco filters, and the resulting precipitate re-dissolved in distilled water giving a clear yellowish solution of monodispersed Pt-decorated CdS NCs with OD 0.6.

Photocatalytic reactions. $\mathrm{H}_{2}$ generation experiments were carried out in a set-up comprising a 5 $\mathrm{cm}$ long cylindrical gas-tight quartz cuvette with two gas outlets, which was filled with $7 \mathrm{~mL}$ liquid leaving a remaining volume of $11.2 \mathrm{~mL}$ Ar into which the evolving $\mathrm{H}_{2}$ escapes. The $7 \mathrm{~mL}$ liquid contains $3.5 \mathrm{~mL} \mathrm{Pt/CdS} \mathrm{(OD} \mathrm{0.6),} \mathrm{Na}_{2} \mathrm{SO}_{3}$, triethanolamine and water. Estimated concentrations of CdS NCs and Pt atoms in this $7 \mathrm{~mL}$ liquid are $0.3 \mu \mathrm{M}$ and $27 \mu \mathrm{M}$, respectively. The reaction mixture is de-aerated under stirring and bubbling with Ar for $30 \mathrm{~min}$ before illumination. The sample was excited in the wavelength range between $350-480 \mathrm{~nm}$ using the filtered output of a 
$450 \mathrm{~W}$ Xe lamp to provide a beam with $1.4 \mathrm{~cm}^{2}$ excitation area and $30 \mathrm{~mW} / \mathrm{cm}^{2}$ excitation power. Aliquots of the gas mixture evolving over the illuminated solution are taken at regular time intervals and analyzed by gas chromatography (Bruker-430-GC) equipped with a thermal conductivity detector. Aliquots of the aqueous mixture are taken for analysis of concentration of $\mathrm{Na}_{2} \mathrm{SO}_{3}$ and TEOA according to ref. 32 and ref. 33, respectively. The external quantum efficiency for $\mathrm{H}_{2}$ generation is determined as the ratio of $2 \times$ number of hydrogen molecules evolved/number of incident photons. The incident power is measured with a thermal power sensor (Thorlabs S302C). The spectrum of the incident light was measured using an Ocean optics USB4000 spectrometer. For an irradiation area of $1.4 \mathrm{~cm}^{2}$ and an irradiation power of $30 \mathrm{~mW} / \mathrm{cm}^{2}$ (approx. photon flux is $482 \mu \mathrm{mol} / \mathrm{h}$ ), $\mathrm{H}_{2}$ production rate of up to $76 \mu \mathrm{mol} / \mathrm{h}$ which corresponds to $31.5 \%$ external quantum efficiency are obtained.

Photocatalyst characterization. Elemental composition of the $\mathrm{Pt} / \mathrm{CdS}$ catalyst was determined with an Inductively Coupled Plasma - Optical Emission Spectrometry (Spectro Ciros ICP-OES). ICP-OES samples were prepared directly from colloidal sample solutions. Morphology and elemental composition of the photocatalyst was further characterized with a JEOL JEM-2100 highangle annular dark field scanning TEM (HAADF-STEM) coupled with energy-dispersive x-ray (EDX) analysis (Oxford, X-Max ${ }^{\mathrm{N}}$ ) and operating at a $200 \mathrm{kV}$ accelerating voltage. Surface ligands of Pt/CdS catalyst were determined with an FT-IR (Bruker, Vertex 80v), equal amount of collected catalysts were pressed into $\mathrm{KBr}$ pellets and mounted into a vacuum chamber, and spectra were recorded with a resolution of $4 \mathrm{~cm}^{-1}$.

Samples for transient absorption measurements exhibited an optical density of 0.5 at $438 \mathrm{~nm}$ in a $2 \mathrm{~mm}$ cuvette, under realistic $\mathrm{H}_{2}$ generation conditions (i.e. $0.1 \mathrm{M}$ TEOA $+0.02 \mathrm{M} \mathrm{Na}_{2} \mathrm{SO}_{3}$, no visible aggregation observed during measurements). Transient absorption measurements 
employed a commercial laser system (Light Conversion: Pharos ultrafast regenerative amplifier based on Yb:KGW lasing medium, Orpheus optical parametric amplifier, and Lyra second harmonic generation unit) producing approximately $200 \mathrm{fs}$ excitation pulses at $350 \mathrm{~nm}(6 \mu \mathrm{W}$ excitation above the CdS band edge) creating around 0.1 excitons per nanocrystal on average. Transient absorption spectra were subsequently acquired with a delayed, low-intensity UV (360 $480 \mathrm{~nm})$ or white light continuum $(450 \sim 750 \mathrm{~nm})$ using a Helios spectrometer (HE-VIS-3200, Ultrafast Systems).

\section{AUTHOR INFORMATION}

\section{Corresponding Author}

*Wei Li, w.li8@aston.ac.uk

*Frank Jäckel, fjaeckel@liv.ac.uk

\section{Author Contributions}

The manuscript was written through contributions of all authors. All authors have given approval to the final version of the manuscript.

\section{ACKNOWLEDGMENT}

Financial support by The University of Liverpool (F.J.) is gratefully acknowledged. The authors further acknowledge support from the EPSRC Laser Loan Pool (EP/G03088X/1). The underlying EPSRC funded data in this paper is available from

http://dx.doi.org/10.17638/datacat.liverpool.ac.uk/208

\section{REFERENCES}


1. Talapin, D.V.; Lee, J.-S.; Kovalenko, M.V.; Shevchenko, E.V. Prospects of colloidal nanocrystals for electronic and optoelectronic applications. Chem. Rev. 2010, 110, 389-458.

2. Hines, D. A.; Kamat, P. V. Recent Advances in Quantum Dot Surface Chemistry. ACS Appl. Mater. Interfaces 2014, 6, 3041-3057.

3. Han, Z.; Qiu, F.; Eisenberg, R.; Holland, P.L.; Krauss, T.D. Robust Photogeneration of $\mathrm{H}_{2}$ in Water Using Semiconductor Nanocrystals and A Nickel Catalyst. Science 2012, 338, 1321-1324.

4. Ben-Shahar, Y.; Scotognella, F.; Kriegel, I.; Moretti, L.; Cerullo, G.; Rabani, E.; Banin, U. Optimal Metal Domain Size for Photocatalysis with Hybrid Semiconductor-Metal Nanorods. Nat. Commun. 2016, 7,10413 .

5. Simon, T.; Bouchonville, N.; Berr, M. J.; Vaneski, A.; Adrovic, A.; Volbers, D.; Wyrwich, R.; Döblinger, M.; Susha, A. S.; Rogach, A. L.; Jäckel, F.; Stolarczyk, J. K.; Feldmann, J. Redox Shuttle Mechanism Enhances Photocatalytic $\mathrm{H}_{2}$ Generation on Ni-Decorated CdS Nanorods. Nat. Mater. 2014, 13, 1013-1018.

6. Berr, M.; Vaneski, A.; Susha, A. S.; Rodríguez-Fernández, J.; Döblinger, M.; Jäckel, F.; Rogach, A. L.; Feldmann, J. Colloidal CdS Nanorods Decorated with Subnanometer Sized Pt Clusters for Photocatalytic Hydrogen Generation. Appl. Phys. Lett. 2010, 97, 093108.

7. Li, W.; O’Dowd, G.; Whittles, T. J.; Hesp, D.; Gründer, Y.; Dhanak, V. R.; Jäckel, F. Colloidal DualBand Gap Cell for Photocatalytic Hydrogen Generation. Nanoscale, 2015, 7, 16606-16610.

8. Das, A.; Han, Z. J.; Haghighi, M. G.; Eisenberg, R. Photogeneration of Hydrogen from Water Using CdSe Nanocrystals Demonstrating the Importance of Surface Exchange. Proc. Natl. Acad. Sci. U.S.A. 2013, $110,16716-16723$.

9. De Roo, J.; Van Driessche, I.; Martins, J. C.; Hens, Z. Colloidal Metal Oxide Nanocrystal Catalysis by Sustained Chemically Driven Ligand Displacement. Nat. Mater. 2016, 15, 517-521.

10. Berr, M. J.; Wagner, P.; Fischbach, S.; Vaneski, A.; Schneider, J.; Susha, A. S.; Rogach, A. L.; Jäckel, F.; Feldmann, J. Hole Scavenger Redox Potentials Determine Quantum Efficiency and Stability of PtDecorated CdS Nanorods for Photocatalytic Hydrogen Generation. Appl. Phys. Lett. 2012, 100, 223903.

11. Wu, K. F.; Chen, Z. Y.; Lv, H. J.; Zhu, H. M.; Hill, C. L.; Lian, T. Q. Hole Removal Rate Limits Photodriven $\mathrm{H}_{2}$ Generation Efficiency in CdS-Pt and CdSe/CdS-Pt Semiconductor Nanorod-Metal Tip Heterostructures. J. Am. Chem. Soc. 2014, 136, 7708-7716.

12. Gimbert-Suriñach, C.; Albero, J.; Stoll, T.; Fortage, J.; Collomb, M.-N.; Deronzier, A.; Palomares, E.; Llobet, A. Efficient and Limiting Reactions in Aqueous Light-Induced Hydrogen Evolution Systems using Molecular Catalysts and Quantum Dots. J. Am. Chem. Soc. 2014, 136, 7655-7661.

13. Chang, C. M.; Orchard, K. L.; Martindale, B. C. M.; Reisner, E. Ligand Removal from CdS Quantum Dots for Enhanced Photocatalytic $\mathrm{H}_{2}$ Generation in $\mathrm{pH}$ Neutral Water. J. Mater. Chem. A, 2016, 4, 2856-2862.

14. Elliott, E. W.; Glover, R. D.; Hutchison, J. E. Removal of Thiol Ligands from Surface-Confined Nanoparticles without Particle Growth or Desorption. ACS Nano, 2015, 9, 3050-3059. 
15. Cho, E. S.; Coates, N. E.; Forster, J. D.; Ruminski, A. M.; Russ, B.; Sahu, A.; Su, N. C.; Yang, F.; Urban, J. J. Engineering Synergy: Energy and Mass Transport in Hybrid Nanomaterials. Adv. Mater. 2015, $27,5744-5752$.

16. Cargnello, M.; Chen, C.; Diroll, B. T.; Doan-Nguyen, V. V. T.; Gorte, R. J.; Murray, C. B. Efficient Removal of Organic Ligands from Supported Nanocrystals by Fast Thermal Annealing Enables Catalytic Studies on Well-Defined Active Phases. J. Am. Chem. Soc. 2015, 137, 6906-6911.

17. Smith, J. G.; Jain, P. K. The Ligand Shell as an Energy Barrier in Surface Reactions on Transition Metal Nanoparticles. J. Am. Chem. Soc. 2016, 138, 6765-6773.

18. Zhao, J.; Holmes, M. A.; Osterloh, F. E. Quantum Confinement Controls Photocatalysis: A Free Energy Analysis for Photocatalytic Proton Reduction at CdSe Nanocrystals. ACS Nano, 2013, 7, 4316-4325.

19. Aldana, J.; Wang, Y.A.; Peng, X. G. Photochemical Instability of CdSe Nanocrystals Coated by Hydrophilic Thiols. J. Am. Chem. Soc. 2001, 123, 8844-8850.

20. Aldeek, F.; Hawkins, D.; Palomo, V.; Safi, M.; Palui, G.; Dawson, P. E.; Alabugin, I.; Mattoussi, H. UV and Sunlight Driven Photoligation of Quantum Dots: Understanding the Photochemical Transformation of the Ligands. J. Am. Chem. Soc. 2015, 137, 2704-2714.

21. Aldana, J.; Lavelle, N.; Wang, Y. J.; Peng, X. G. Size-Dependent Dissociation pH of Thiolate Ligands from Cadmium Chalcogenide Nanocrystals. J. Am. Chem. Soc. 2005, 127, 2496-2504.

22. Wang, Y. A.; Li, J. J.; Chen, H. Y.; Peng, X. G. Stabilization of Inorganic Nanocrystals by Organic Dendrons. J. Am. Chem .Soc. 2002, 124, 2293-2298.

23. Yu, W. W.; Qu, L. H.; Guo, W. Z.; Peng, X. G. Experimental Determination of the Extinction Coefficient of CdTe, CdSe, and CdS Nanocrystals. Chem. Mater. 2003, 15, 2854-2860.

24. Brannon, D.G.; Morrison, R. H.; Hall, J. L.; Humphrey, G. L.; Zimmerman, D. N. Spectra and bonding for copper(II)-aminoalcohol complexes I: The I.R. Spectra of Complexes of Mono-, Di- and Triethanolamine. J. Inorg. Nucl. Chem. 1971, 33, 981-990.

25. Min'kov, V. S.; Chesalov, Y. A.; Boldyreva, E. V. Study of the Temperature Effect on IR Spectra of Crystalline Amino Acids, Dipeptides, and Polyamino acids. IV. L-Cysteine and DL-Cysteine. J. Struct. Chem. 2008, 49, 1022-1034.

26. Li, Y. X.; Zhang, K.; Peng, S. Q.; Lu, G. X.; Li, S. B. Photocatalytic Hydrogen Generation in the Presence of Ethanolamines over $\mathrm{Pt} / \mathrm{ZnIn}_{2} \mathrm{~S}_{4}$ under Visible Light Irradiation. J. Mol. Catal. A: Chem. 2012, $363,354-361$.

27. Klimov, V. I. Optical Nonlinearities and Ultrafast Carrier Dynamics in Semiconductor Nanocrystals. $J$. Phys. Chem. B 2000, 104, 6112-6123.

28. Berr, M. J.; Vaneski, A.; Mauser, C.; Fischbach, S.; Susha, A. S.; Rogach, A. L.; Jäckel, F.; Feldmann, J. Delayed Photoelectron Transfer in Pt-Decorated CdS Nanorods under Hydrogen Generation Conditions. Small 2012, 8, 291-297.

29. Wu, K. F.; Zhu, H. M.; Lian, T. Q. Ultrafast Exciton Dynamics and Light-Driven $\mathrm{H}_{2}$ Evolution in Colloidal Semiconductor Nanorods and Pt-Tipped Nanorods. Acc. Chem. Res. 2015, 48, 851-859. 
30. Yu, W. W.; Peng, X. G. Formation of High-Quality CdS and Other II \pm VI Semiconductor Nanocrystals in Noncoordinating Solvents: Tunable Reactivity of Monomers. Angew. Chem. Int. Ed. 2002, 41, 2368-2371.

31. Tamang, S.; Beaune, G.; Texier, I.; Reiss, P. Aqueous Phase Transfer of InP/ZnS Nanocrystals Conserving Fluorescence and High Colloidal Stability. ACS Nano, 2011, 5, 9392-9402.

32. Yang, Z. H.; Zhang, Y. X.; Meng, Z. Q.; Zhang, Q. X. UV-Visible Absorption Spectral Properties of Sulfur Dioxide and Its Derivatives. Environ. Chem. 2013, 32, 188-194.

33. Fleck, H. R. The Detection and Determination of Triethanolamine, Analyst, 1935, 60, 77-79. 
Table of Contents Graphic

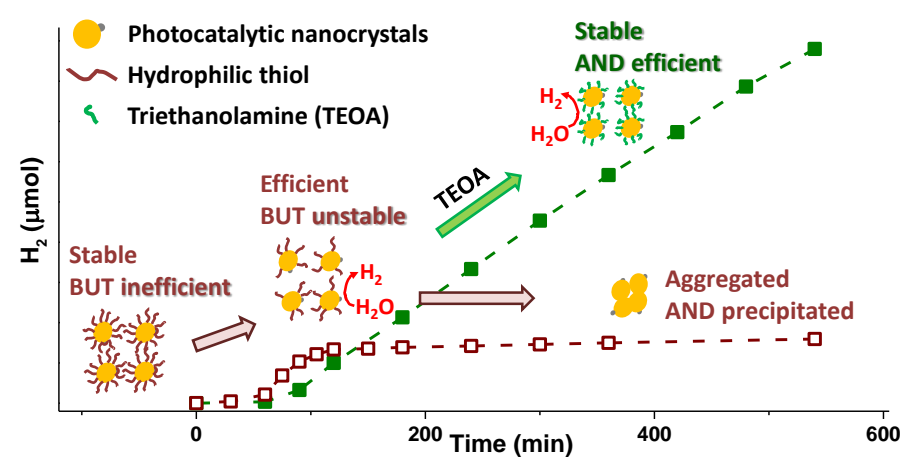

